\title{
Amygdalin isolated from Amygdalus mongolica protects against hepatic fibrosis in rats
}

\author{
JIA WANG ${ }^{1,2, a}$ \\ HONGBING ZHOU 1,2,a \\ TONG WU $\mathrm{W}^{1,3}$ \\ PEISAI WU ${ }^{1,4}$ \\ QUANLI LIU ${ }^{2}$ \\ SONGLI SHI ${ }^{1, *}$ \\ ${ }^{1}$ Department of Pharmacy, Baotou \\ Medical College, Baotou, Inner Mongolia \\ China, 014040 \\ ${ }^{2}$ Institute of Bioactive Substance and \\ Mongolian Medicine and Chinese Materia \\ Medica, Baotou, Inner Mongolia, China \\ 014040 \\ ${ }^{3}$ The Third Staff Hospital of Baogang \\ Group, Baotou, Inner Mongolia, China \\ 014010 \\ ${ }^{4}$ Hunan University of Medicine, Huaihua \\ Hunan, China, 418000
}

Accepted July 21, 2020

Published online August 26, 2020

\begin{abstract}
The aim of this research was to investigate the effect of amygdalin on hepatic fibrosis in rats. Amygdalin was purified and identified from the seeds of Amygdalus mongolica. Sprague Dawley rats in the control and model groups were administered water. Sprague Dawley rats were divided into the low-, middle-, and high-dose amygdalin groups that received 20, 40, and $80 \mathrm{mg} \mathrm{kg}^{-1}$ amygdalin, respectively. whereas the silymarin group was treated with $50 \mathrm{mg}$ $\mathrm{kg}^{-1}$ silymarin. The control and model groups were administered water. Liver tissue analysis revealed significantly lower activities of ALT, AST, ALP, SOD, and MDA in the drug-treated groups compared to the model group. Serum analysis revealed significantly lower HYC and C-IV in the middle-dose amygdalin-treated group compared to the model group. The histopathological changes were less severe in the drug-treated groups as observed by the formation of pseudolobuli and decreased collagen fiber deposition. Hepatic fibrosis-related genes were expressed at significantly lower levels in the amygdalin-treated groups than in the model group. Amygdalin from A. mongolica represents a therapeutic candidate for hepatic fibrosis prevention and treatment.
\end{abstract}

Keywords: amygdalin, Amygdalus mongolica, hepatic fibrosis, rat model, traditional Chinese medicine

Liver fibrosis occurs in response to a variety of insults, especially chronic injuries including viral hepatitis, alcohol abuse, drug use, autoimmune attack of hepatocytes or bile duct epithelial cell, or congenital abnormalities (1). Both quantitative and qualitative changes in the composition of the hepatic extracellular matrix happen as the liver becomes fibrotic. Hepatic fibrosis can lead to irreversible cirrhosis, which involves multiple cellular and molecular events (2). If properly treated at the fibrotic stage, cirrhosis can be prevented. However, if the treatment is not timely, the disorder can even lead to liver cancer (3). Although the antifibrotic activities of many compounds have been demonstrated in animal models, none of these compounds has been thoroughly validated in the clinic or commercialized as a therapy for fibrosis (4). Amygdalin is one of the active constituents of Semen

* Correspondence; e-mail: shisongli122@126.com;

a These authors contributed equally to this work. 
armeniacae, a traditional Chinese medicine. Studies have found that this cyanogenic glycoside compound is widely used to inhibit fibrosis of the lungs, kidneys, and liver $(5,6)$.

Amygdalin can be found in a variety of plant species, specifically in rosaceous plant seeds, including apricot, cherry, plum, and peach seeds (7). Amygdalus mongolica (Maxim.) Ricker (Rosaceae) is a native plant of the Inner Mongolia plateau where it is known as Wulian-Buyilesi $(8,9)$. A. mongolica contains various nutrients including flavonoids, proteins, fats, phenols, organic acids, polysaccharides, and vitamin E, all of which are also used the Chinese medicine $(10,11)$. Numerous studies have documented that amygdalin possesses the ability to induce cell apoptosis. Amygdalin has been used to treat different types of cancers and organ fibrosis. Guo et al. (12) unequivocally determined that amygdalin is a potent antifibrotic agent that may have therapeutic potential for patients with fibrotic kidney diseases. Luo et al. (13) elucidated the mechanism by which amygdalin reduces liver fibrosis; its role in regulating the expression of platelet-derived growth factor has been investigated. Hepatic fibrosis is considered to be a major global health issue due to the high morbidity, severe economic burden, and psychological pressure that it causes. Because there is still a lack of effective drugs and therapeutic strategies, this study examined the antifibrotic effect of amygdalin from A. mongolica seeds on hepatic fibrosis.

\section{EXPERIMENTAL}

\section{Materials}

The seeds of A. mongolica were collected from the Alashan Yabrai Gobi in Inner Mongolia in June 2016. The dry, mature seeds were identified by Professor Songli Shi of Baotou Medical College. The seeds of A. mongolica were placed in a drying oven at $55^{\circ} \mathrm{C}$ for $8 \mathrm{~h}$ followed by cleaning, shelling, and crushing.

High-purity grade amygdalin was purchased from (Shanghai, China). The silymarin capsules were purchased from Madaus AG (Germany). The malondialdehyde (MDA) kit, superoxide dismutase (SOD) kit, hydroxyproline (HYP) kit, alanine aminotransferase (ALT) kit, aspartate aminotransferase (AST) kit, alkaline phosphatase (ALP) kit, Masson kit, and total protein quantitative assay kit were purchased from Jiancheng Biology Engineering Institute (China). The hyaluronic acid (HA) kit, laminin (LN) kit, procollagen III (PC-III) kit, and collagen IV (C-IV) kit were purchased from Beijing North Institute of Biotechnology (China). The TRIzol kit, RevertAid First Strand cDNA Synthesis Kit, and Real SYBR Mixture kit were purchased from Thermo Fisher Scientific (China).

\section{Isolation and identification of amygdalin in A. mongolica}

Amygdalin was isolated from A. mongolica seeds for the first time in our laboratory (14). Crushed A. mongolica seeds were dissolved in $95 \%$ ethanol $\left(1: 10 \mathrm{~g} \mathrm{~mL}^{-1}\right)$ and extracted using a Soxhlet apparatus three times for $2 \mathrm{~h}$ each. A rotary evaporator and water bath were used to concentrate the crude extract to an oily residue. According to the solvent polarity by liquid/liquid extraction, petroleum ether, ethyl acetate, $n$-butanol, and water were successively used to extract the less polar substances from the oily residue. The yield of the $n$-butanol extraction sites to the oily residue was $41.87 \%$.

The $n$-butanol extraction sites were applied to a D101 macroporous resin column and eluted with 10, 30, 50, 70, and $90 \%$ ethanol. Thin-layer silica gel chromatography was per- 
formed to assess the elution steps. Carbon tetrachloride and methanol were combined (4:1) as the developing solvent. The 10 and $30 \%$-alcohol eluates were loaded on an octadecylsilyl (ODS) column for gradient elution with methanol (10, 20, 30, 40, 50, 60, 70, 80, 90, and $100 \%$ ). The developing solvent contained ethyl acetate, formic acid, and water (20:3:1). Finally, a colorless powder designated compound I was obtained by pooling the fluxes with the same chromatographic characteristics and recovering the solvent.

The structural identification of the colorless powder as compound I was performed by the Inner Mongolia Key Laboratory of Fine Organic Synthesis at Inner Mongolia University. The ultra-performance liquid chromatography (UPLC) conditions were as follows: Agilent ZORBAX Eclipse Plus $\mathrm{C}_{18}$; the mobile phase A was $0.1 \%$ formic acid in aqueous solution, the mobile phase $\mathrm{B}$ was methanol, and the flow rate was $0.4 \mathrm{Ml} \mathrm{min}{ }^{-1}$; the elution gradient was $0-3 \mathrm{~min} 10-30 \%$ B, 4-5 $\min 30-100 \%$ B, 5-6 min $100 \%$ B. The electron spray ionizationmass spectrum (ESI-MS) conditions were as follows: negative ion mode; debris voltage of $50 \mathrm{~V}$; capillary voltage of $3500 \mathrm{~V}$; atomization pressure of $45 \mathrm{psi}$; drying gas temperature of $350{ }^{\circ} \mathrm{C}$; mass spectrometry ion range of $100-1500 \mathrm{~m} / \mathrm{z}$. The nuclear magnetic resonance (NMR) conditions were as follows: $\mathrm{D}_{2} \mathrm{O}$ as the solvent, frequency at $500 \mathrm{MHz}$.

\section{Animals and hepatic fibrosis model establishment}

Sixty male Sprague Dawley (SD) rats, SPF grade, bodyweight 170-200 g, were purchased from the Peking University Health Science Center (Laboratory Animal Science Department), license No. 2011-0012 (Beijing) SCXK and used in the hepatic fibrosis experiments. All rats were housed in an animal room at a controlled temperature of $25 \pm 2{ }^{\circ} \mathrm{C}$ and a relative humidity of $55 \%$. All procedures involving rats were approved by the Committee on the Ethics of Animal Experiments of the Institute, Baotou Medical College, Baotou, Inner Mongolia, China. All efforts were made to minimize animal suffering.

The animals were fed a basic diet for one week to adapt to the new living environment. Subsequently, the rats were randomly divided into 6 groups (the Control, Model, Low-Dose, Middle-Dose, High-Dose, and Silymarin groups) with 10 rats/group.

The rat hepatic fibrosis model was established as previously described (15-17). The rats received subcutaneous injections of carbon tetrachloride $\left(\mathrm{CCl}_{4}\right), 5 \mathrm{~mL} \mathrm{~kg}^{-1}$ as an initial dose and $3 \mathrm{~mL} \mathrm{~kg}^{-1}$ as subsequent doses, dissolved in $40 \%$ olive oil 2 times per week for

Table I. Body mass, liver/spleen ratio of rats in different groups

\begin{tabular}{lcc}
\hline Group $^{\mathrm{a}}$ & Body mass $(\mathrm{g})$ & Liver/Spleen ratio \\
\hline Control & $438.86 \pm 14.75$ & $14.82 \pm 0.94$ \\
\hline Model & $339.83 \pm 22.58^{*}$ & $24.52 \pm 1.82^{*}$ \\
\hline Low-dose & $365.86 \pm 21.45^{* \#}$ & $19.16 \pm 2,70^{* \#}$ \\
\hline Middle-dose & $392.67 \pm 24.55^{* \#}$ & $23.64 \pm 2.76^{*}$ \\
\hline High-dose & $352.50 \pm 21.45^{*}$ & $22.80 \pm 3.37^{*}$ \\
Silymarin & $372.33 \pm 24.67^{* \#}$ & $22.95 \pm 1.79^{*}$ \\
\hline
\end{tabular}

a The number of rats in each group was 10; ${ }^{*}$ significant from control $p<0.05 ;{ }^{\sharp}$ significant from model $p<0.05$. 
8 weeks. The rats in the control group without $\mathrm{CCl}_{4}$ treatment remained healthy during this period. In the $\mathrm{CCl}_{4}$ treatment groups, the body mass was significantly decreased but the liver/spleen ratio was significantly increased compared to the corresponding values in the untreated control group (Table I). Therefore, a rat model of hepatic fibrosis was successfully established.

\section{Drug delivery, serum parameter measurements, and liver histopathological examination}

The $L D_{50}$ (lethal dose causing $50 \%$ survival in a group) of amygdalin was determined in a series of five experiments, each using 60 rats. The amygdalin doses ranged from below $400 \mathrm{mg}$ per $\mathrm{kg}$ of body weight to above 1,100 mg per $\mathrm{kg}$ of body mass. The results showed that the $L D_{50}$ of amygdalin was approximately $522 \mathrm{mg} \mathrm{kg}^{-1}(18)$. Three groups received 20, $40,80 \mathrm{mg} \mathrm{kg}^{-1}$ amygdalin as the low-, middle-, or high-dose, respectively, whereas one comparator-treated group received silymarin $\left(50 \mathrm{mg} \mathrm{kg}^{-1}\right)$. The treatments were administered by gavage of $5 \mathrm{~mL} \mathrm{~kg}^{-1}$ amygdalin or silymarin solution into the stomach. The low-, middle-, and high-amygdalin dose groups were treated with 4, 8, and $16 \mathrm{mg} \mathrm{mL}^{-1}$ amygdalin, respectively. The concentration of the silymarin solution was $10 \mathrm{mg} \mathrm{mL}^{-1}$. The rats in the model group and the control group received $5 \mathrm{~mL} \mathrm{~kg}^{-1}$ distilled water. Treatments were performed 2 times a week for 8 weeks.

After the intraperitoneal injection of $10 \%$ chloral hydrate, blood samples were drawn from the abdominal aorta into heparinized injectors and centrifuged at 6,000 rpm for 10 min at $4{ }^{\circ} \mathrm{C}$. Liver function indexes, including ALT, AST, ALP, HYP, HA, LN, PC-III, C-IV, SOD, and MDA, were detected in the serum or liver tissues according to the instructions of the respective kits.

After finishing the blood collection, liver samples were dissected, washed with ice-cold saline, and immediately stored at $-80^{\circ} \mathrm{C}$ until further analysis. Partial liver samples $(1.0 \times 1.0 \times 0.3 \mathrm{~cm}$ liver tissue from the middle part of the right lobe of the liver) from each rat were fixed in $10 \%$ neutral formalin and embedded with paraffin. All the specimens were sectioned at thicknesses of $4 \mu \mathrm{m}$. To conduct the histopathology analysis, hematoxylin and eosin (H\&E) and Masson's staining were used. Images of the histopathological samples from each group were examined by a pathologist.

\section{RNA isolation and quantitative real-time PCR (RT-PCR) analysis}

Total RNA was extracted from liver tissue using the TRIzol kit according to the manufacturer's instructions. The gene-specific primers were designed using Primer Premier 5.0 software (Table II). The reverse transcription reactions were performed with the Revert

Table II. The primer of $q R T-P C R$

\begin{tabular}{lcc}
\hline Gene Name & Forward $\left(5^{\prime}-3^{\prime}\right)$ & Reverse $\left(5^{\prime}-3^{\prime}\right)$ \\
\hline RAT Actin & CCCATCTATGAGGGTTACGC & TTTAATGTCACGCACGATTTC \\
TGF- $\beta 1$ & CGCAACAACGCAATCTATG & ACCAAGGTAACGCCAGGA \\
Smad3 & CCAGTGCTACCTCCAGTGTT & CTGGTGGTCGCTAGTTTCTC \\
Smad7 & GGCTTTCAGATTCCCAACTTC & CGCCATCCACTTCCCTTGT \\
\hline
\end{tabular}


Aid First-Strand cDNA Synthesis Kit following the manufacturer's instructions. RT-PCR was performed with a 7900HT RT-PCR instrument (ABI company) using the Real SYBR Mixture kit. The thermal cycling conditions were as follows: initial denaturation at $95{ }^{\circ} \mathrm{C}$ for $35 \mathrm{~s}$, then 40 cycles of denaturation at $95^{\circ} \mathrm{C}$ for $5 \mathrm{~s}$, followed by annealing and extension at $60^{\circ} \mathrm{C}$ for $20 \mathrm{~s}$. Quantitative expression changes were calculated using the $2^{-\Delta \Delta \mathrm{CT}}$ method.

\section{Statistical analysis}

The experimental data were examined using SPSS 17.0 software for statistical data processing. To determine statistically significant differences, the data were subjected to one-way analysis of variance (ANOVA) and the least significant difference (LSD) post hoc test. The criterion for significance was set at $p<0.05$.

\section{RESULTS AND DISCUSSION}

\section{Compound structure identification}

The main constituent of compound I was identified as amygdalin by UPLC-ESI-MS and NMR. The UPLC chromatogram showed that the $n$-butanol extraction sites had a peak at a retention time of $2.2 \mathrm{~min}$. The product-ion spectra from the ESI-MS analysis identified the following product-ions: $[\mathrm{M}-\mathrm{H}]^{-}, m / z 456 ;[\mathrm{M}+\mathrm{COOH}]^{-}, m / z 502 ;$ and $\left[\mathrm{M}_{-} \mathrm{C}_{2} \mathrm{H}_{2}\right]^{-}, \mathrm{m} / z 431$. The results characterized compound I as a small molecule with a relative molecular mass of 457, suggesting that it is a cyanogenic glycoside (Fig. 1).

a)

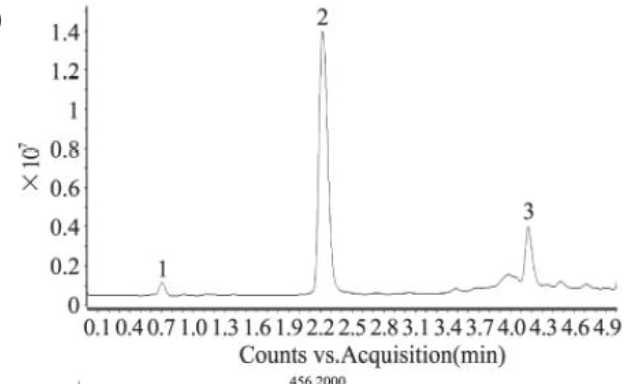

b)

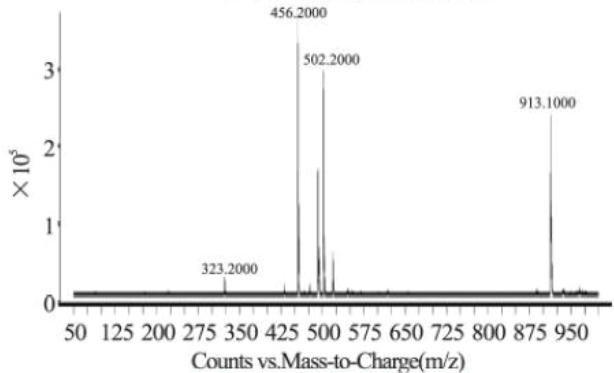

Fig. 1. UPLC analysis of the purified extract: a) UPLC chromatogram of the purified extract; b) ESI-MS spectra of compound I. 
Table III. ${ }^{13} \mathrm{C}$ NMR data of compound I

\begin{tabular}{|c|c|c|}
\hline Ring-C Number & Number & Chemical shift (ppm) \\
\hline \multirow{8}{*}{$\mathrm{A}$} & 1 & 132.38 \\
\hline & 2 & 129.32 \\
\hline & 3 & 127.70 \\
\hline & 4 & 130.44 \\
\hline & 5 & 127.83 \\
\hline & 6 & 129.28 \\
\hline & 7 & 68.44 \\
\hline & 8 & 118.50 \\
\hline \multirow{6}{*}{ B } & 9 & 101.45 \\
\hline & 10 & 72.58 \\
\hline & 11 & 75.83 \\
\hline & 12 & 69.59 \\
\hline & 13 & 75.42 \\
\hline & 14 & 69.08 \\
\hline \multirow{6}{*}{$\mathrm{C}$} & 15 & 102.96 \\
\hline & 16 & 73.12 \\
\hline & 17 & 75.81 \\
\hline & 18 & 69.52 \\
\hline & 19 & 75.60 \\
\hline & 20 & 60.67 \\
\hline
\end{tabular}

The ${ }^{1} \mathrm{H}$ NMR analysis $\left(\mathrm{D}_{2} \mathrm{O}, 500 \mathrm{MHz}\right)$ data showed monosubstituted benzene ring signals at $\delta 7.43(2 \mathrm{H}, \mathrm{m}, \mathrm{H}-2,6), 7.36(3 \mathrm{H}, \mathrm{m}, \mathrm{H}-3,4,5)$, a continuous oxygen methylene signal at $\delta 5.89(1 \mathrm{H}, \mathrm{s}, \mathrm{H}-7)$, and two molecular glucose signals at $\delta 4.65(1 \mathrm{H}, \mathrm{d}, J=8.1 \mathrm{~Hz}, \mathrm{H}-15)$ and $4.62(1 \mathrm{H}, \mathrm{d}, J=7.5 \mathrm{~Hz}, \mathrm{H}-9)$. The ${ }^{13} \mathrm{C}$ NMR spectrum data indicated the presence of three rings (Table III). The NMR data were consistent with the reported characterization of amygdalin, indicating that the compound I preparation contains amygdalin.

Solid crystallization was obtained by combining the flow fraction containing the same composition and recovering the solvent. Finally, the content of amygdalin in the $n$-butanol extraction sites of $A$. mongolica was $47.72 \%$.

\section{Effects of amygdalin on the activities of ALT, AST, and ALP}

Hepatic fibrosis is a reversible pathological process that acts as a repair response to chronic liver injuries (19). Li et al. (20) found that the increase in ALT, AST, and ALP in the serum indicates severe liver damage. Measurements of the activity levels of ALT, AST, and ALP, which are serum markers of liver function, indicated that these three enzymes 

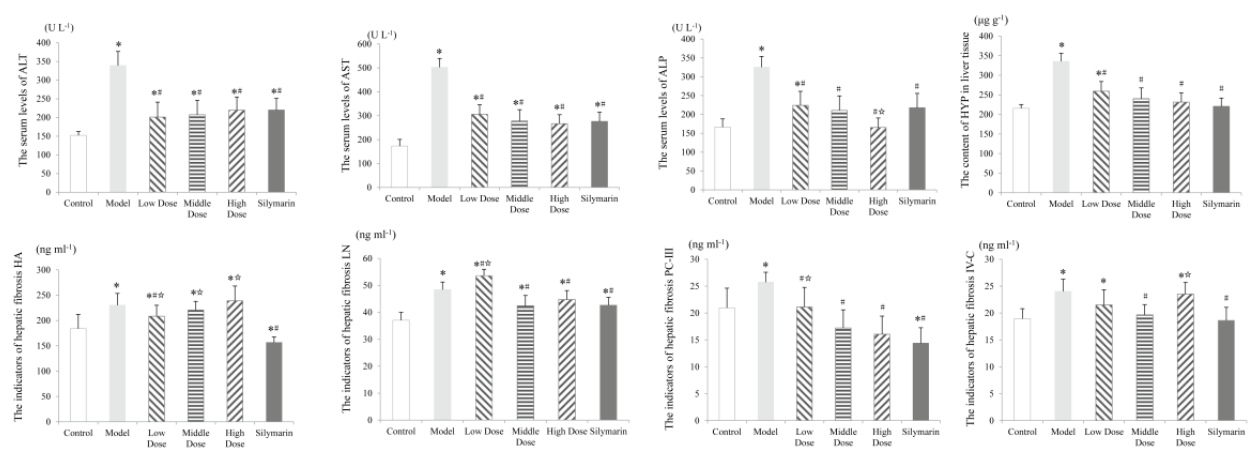

Fig. 2. Liver function indexes in the serum or liver tissues: * significant from control $p<0.05$; \# significant from model $p<0.05$; significant from silymarin $p<0.05$.

exhibited higher activities in the model group than in the control group (Fig. 2). As indicators of drug efficacy in this animal model, the activities of these three enzymes were significantly decreased in the groups treated with silymarin and different doses of amygdalin, compared with those in the model group $(p<0.05)$. Compared with that in the silymarin group, only the ALP value in the high-dose group showed a significant difference $(p<0.05)$, and the other indexes showed no significant difference. Therefore, amygdalin from A. mongolica alleviated the extent of hepatic fibrosis to varying degrees.

\section{Effects of amygdalin on the content of HYP}

It was reported that elevated HYP levels had the best potential for predicting liver fibrosis (21). Therefore, the HYP level was another critical index for evaluating hepatic fibrosis. As shown in Fig. 2, the level of HYP was higher in the model group than in the control group $(p<0.05)$. Detection after drug treatment indicated that the HYP level was significantly decreased in the three amygdalin-treated groups compared with the model group $(p<0.05)$. However, only the middle- and high-dose groups did not differ significantly from the control group. A comparison between the drug administration group and the positive drug-treated group revealed that there was no significant difference in the level of HYP. This result might indicate that amygdalin and silymarin had the same effect on liver fibrosis in rats.

\section{Effects of amygdalin on the liver fibrosis indexes HA, LN, PC-III, and IV-C}

The stages of hepatic fibrosis are correlated with the serum levels of HA, LN, PC-III, and IV-C, which are markers that are used to detect the degree of hepatic fibrosis (22). It has been reported that HA is a major resource for the extracellular matrix. PC-III is a marker used in the diagnosis of hepatic fibrosis and early cirrhosis (23). The serum levels of LN and IV-C are indexes of the rate of basement membrane turnover and indicate the degree of fibrosis in the portal area and sinusoidal capillaries (24). The results of these four indicators are shown in Fig. 2. The serum levels of the liver fibrosis indictors HA, LN, PC-III, and IV-C were significantly increased in the model group compared with the control group $(p<0.05)$. The 
indexes of HA and IV-C were significantly decreased by amygdalin in the middle-dose group compared to the model group $(p<0.05)$. Furthermore, the LN level was significantly lower in the middle- and high-dose amygdalin-treated groups than in the model group $(p<$ 0.05). Only the PC-III indicator values were significantly decreased by the three doses of amygdalin in the treatment groups, compared to the model group $(p<0.05)$. Furthermore, the PC-III indicator values of the amygdalin treatment groups did not significantly differ from those of the control group. A comparison between the drug administration group and the positive drug group showed that the serum levels of the liver fibrosis indictor HA showed significant differences at all three doses $(p<0.05)$. All these results showed that amygdalin from $A$. mongolica alleviated the extent of hepatic fibrosis to varying degrees.

\section{Effects of amygdalin on the activity of SOD and MDA}

Oxidative stress is related to liver fibrosis and precedes hepatic stellate cells (HSC) activation (25). The alteration in the MDA and SOD levels in the liver and serum is considered an index of collagen metabolism and provides valuable information about the biochemical and pathological events in hepatic fibrosis (26). To evaluate the degree of liver damage, we detected the activities of SOD and MDA in the serum and liver tissues. As shown in Fig. 3, the SOD activity in the serum and liver tissue was significantly lower in the model group than in the control group. Assessing the drug efficacy based on this model, the different
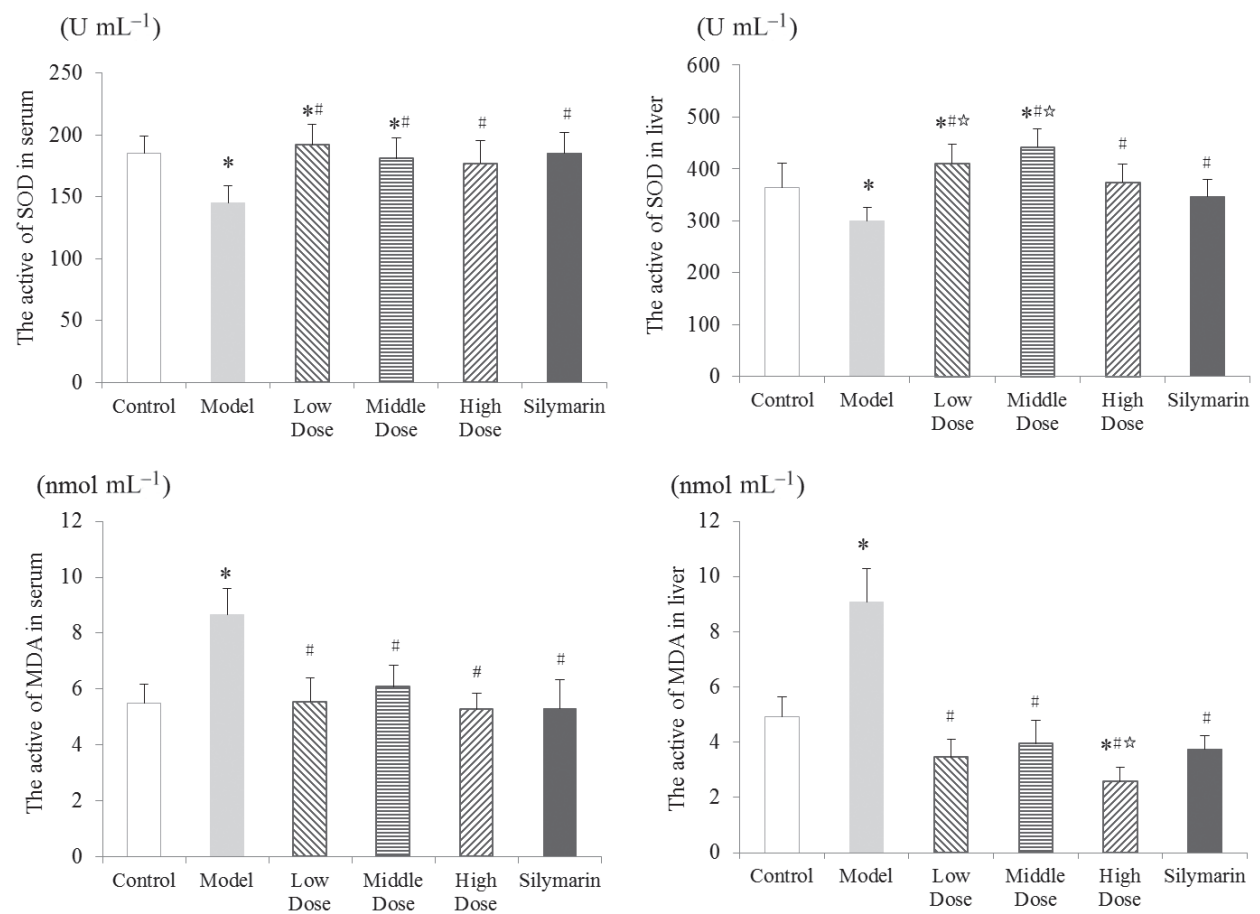

Fig. 3. The active of SOD and the content of MDA in serum and liver: ${ }^{*}$ significant from control $p<$ 0.05 ; \# significant from model $p<0.05$; significant from silymarin $p<0.05$. 
amygdalin doses significantly increased the SOD activity levels in the serum and liver tissues of the treatment groups, compared with those in the model group. However, only the high-dose group differed significantly from the model group $(p<0.05)$, and it was not significantly different from the control group. As shown in Fig. 3, the MDA levels in the serum and liver tissue samples of the model group were significantly increased compared to those in the serum and liver samples of the control group $(p<0.05)$. Detection after drug treatment indicated that the serum and liver samples of the low-, middle-, and high-dose amygdalin-treated groups and the silymarin-treated group had significantly decreased MDA levels compared with those of the model group. Compared with those in the silymarin group, the activities of SOD and MDA in the serum showed no significant differences. However, the activity of SOD in the low- and middle-dose groups and MDA in the high-dose group showed significant differences in the liver tissues $(p<0.05)$. All these results indicate that amygdalin from $A$. mongolica alleviated hepatic fibrosis by regulating the activities of SOD and MDA in the antioxidant system.

\section{Effect of amygdalin on liver pathology in rats with hepatic fibrosis}

The histopathological analysis by H\&E staining indicated different degrees of fibrosis in the liver tissues except in those of the control group. However, there were significant differences between the drug treatment groups and the model group (Fig. 4). We detected

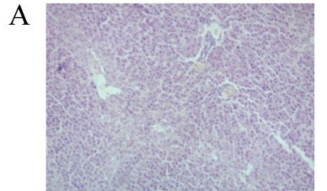

a

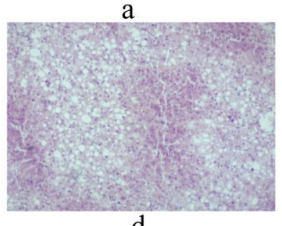

d

B
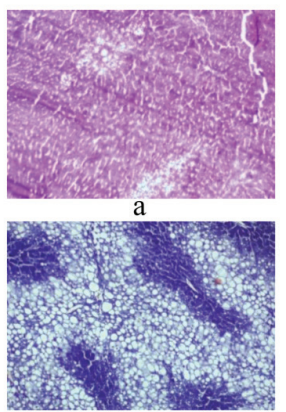

d

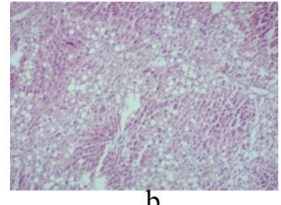

b

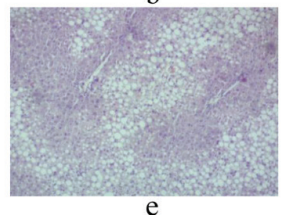

e

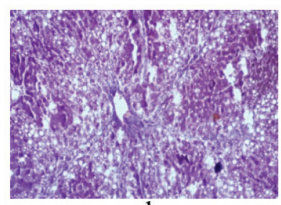

b

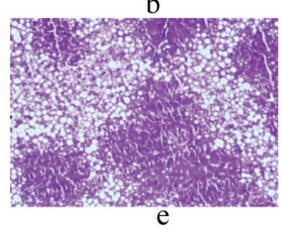

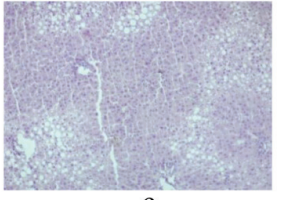

c
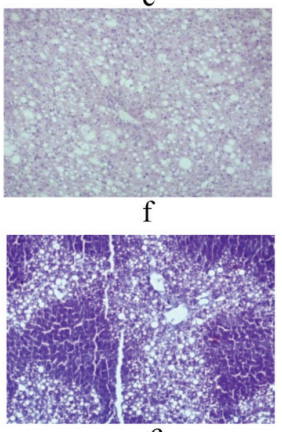

C

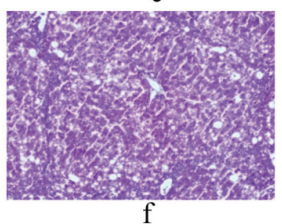

Fig. 4. Effect of amygdalin on liver pathology in rats with hepatic fibrosis). A: HE staining, 100-fold; B: Masson's staining, 100-fold; a) control group, b) model group, c) low dose group, d) middle dose group, e) high dose group, f) silymarin group. 
many liver abnormalities in the model group, including the destruction or disappearance of lobular structures, large deposits of collagen fibers in the catheter area and interlobular area, liver lobules resegmented by fibrous strands, the formation of nodular pseudolobuli, severe steatosis in hepatocytes complicated with moderate edema, and necrosis (Fig. 4A-b). The histopathological changes were alleviated in the drug-treated groups, as observed by pseudolobuli formation and decreased collagen fiber deposition compared to those features in the model group (Fig. 4A-c,d,e,f).

Histopathological analysis by Masson staining showed that the control group had the following characteristics: hepatocytes arranged radially around the central vein, normal structure of hepatic lobules and hepatocytes, and only a small amount of collagen fibers present in the portal area (Fig. 4B-a). In contrast, there were many liver abnormalities in the model group, including the destruction or disappearance of hepatic lobules, large deposits of collagen fibers in the catheter area and interlobular area, and formation of nodular pseudolobuli (Fig. 4B-b). The observed histopathological abnormalities of the model group were alleviated in the drug-treated groups, as observed by pseudolobuli formation and decreased collagen fiber deposition (Fig. 4B-c,d,e,f).

All the results of the liver histopathological analysis showed that HE and Masson's trichrome staining revealed normal liver tissues with distinct hepatic lobules and pseudolobuli. The liver tissue structure in the model group was disordered, and the liver tissues were replaced by a large amount of connective tissue. However, significant improvement was observed in the drug-treated groups compared to the model group.
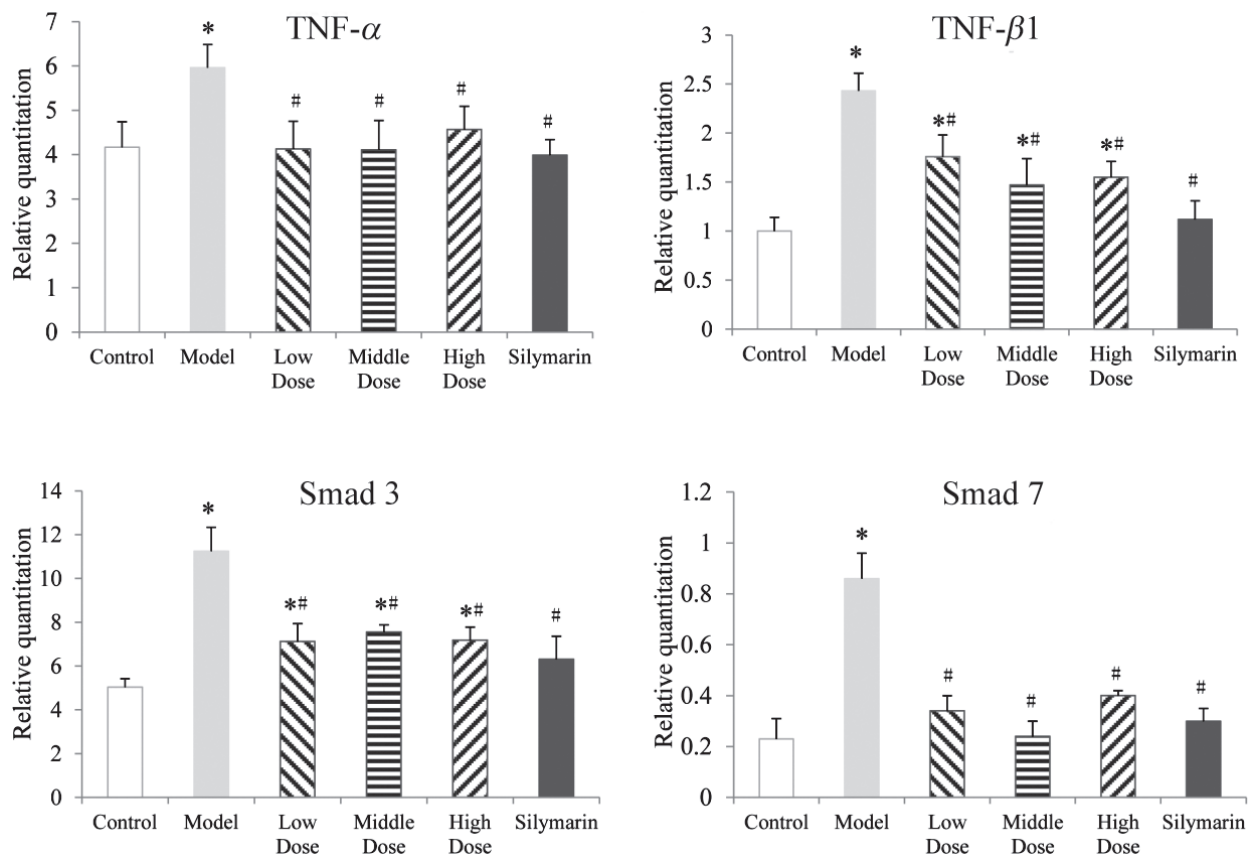

Fig. 5. The expression of TNF- $\alpha$, TGF- $\beta 1$, Smad 3, and Smad 7: ${ }^{*}$ significant from control $p<0.05$; \# significant from model $p<0.05$. 


\section{Differential expression analysis of the hepatic fibrosis-related genes TNF- $\alpha$, TGF- $\beta$, Smad 3, and Smad 7}

Various chemokines are critical mediators of progressive liver damage and fibrosis development $(27,28)$. Tumor necrosis factor $\alpha(T N F-\alpha)$ is a cytokine-induced by fibrosis, and its expression levels are positively correlated with the degree of fibrosis (29). Previous findings have shown that TGF- $\beta$ and the Smad signal transduction pathway are closely associated with the genesis and development of hepatic fibrosis $(30,31)$. As shown in Fig. 5 , the expression of TNF- $\alpha$ was significantly increased in the model group compared to the control group $(p<0.05)$. Administration of different doses of amygdalin significantly reduced the expression levels of TNF- $\alpha$ in the treatment groups compared with those in the model group $(p<0.05)$. Smad proteins transduce signals from TGF- $\beta$ to regulate cell proliferation, differentiation, and cell death activating of receptor serine/threonine kinases. As shown in Fig. 5, the expression levels of TGF- $\beta$, Smad 3, and Smad 7 were significantly increased in the model group, compared to the control group $(p<0.05)$. Importantly, each amygdalin dose significantly decreased the expression levels of TGF- $\beta$, Smad 3, and Smad 7 compared with those in the model group $(p<0.05)$. Therefore, these data showed at the molecular level that amygdalin from A. mongolica regulated the process of hepatic fibrosis.

\section{CONCLUSIONS}

In this study, amygdalin was isolated from A. mongolica seeds and identified. The antifibrotic effect of amygdalin on hepatic fibrosis induced by $\mathrm{CCl}_{4}$ in rats was demonstrated. The histopathological data showed that amygdalin from A. mongolica had a significant protective effect against hepatic fibrosis. This study provides scientific guidance for the further development of amygdalin from A. mongolica as a potential therapeutic agent in the prevention and treatment of hepatic fibrosis.

Acknowledgements. - This research was supported by grants from the National Natural Science Foundation of China (NO. 81641137, 81760782), the Inner Mongolia Natural Science Foundation Project (NO. 2018LH03028; 2019MS08189), the Inner Mongolia autonomous region higher school youth scientific talents support project (NO. NJYT-15-B01), the Inner Mongolia Autonomous Region "Grassland Talents" Project Young Innovative and Entrepreneurial Talents Project (NO. Q2017046), the Doctor Foundation of Baotou Medical College (NO. BSJJ201810), and the Pharmacokinetics and Safety Evaluation research of Mongolian Medicines (Inner Mongolia Science \& Technology Department (2016)66 and Baotou Science and Technology Bureau Intellectual Property (2016)91).

\section{REFERENCES}

1. S. L. Friedman, Molecular regulation of hepatic fibrosis, an integrated cellular response to tissue injury, J. Bio. Chem. 4 (2000) 2247-2250; https://doi.org/ 10.1074/jbc.275.4.2247

2. R. T. Hong, J. M. Xu and Q. Mei, Melatonin ameliorates experimental hepatic fibrosis induced by carbon tetrachloride in rats, World J. Gastroenterol. 12 (2009) 1452-1458; https://doi.org/10.3748/ wjg.15.1452

3. M. Pinzani and K. Rombouts, Liver fibrosis: from the bench to clinical targets, Dig. Liver Dis. 4 (2004) 231-242; https://doi.org/10.1016/j.dld.2004.01.003

4. T. Christian, L. F. Scott, S. Detlef and P. Massimo, Hepatic fibrosis: Concept to treatment, J. Hepatol. 1 (2015) S15-24; https://doi.org/10.1016/j.jhep.2015.02.039 
5. H. K. Du, F. C. Song, X. Zhou, H. Li and J. P. Zhang, Effect of amygdalin on serum proteinic biomarker in pulmonary fibrosis of bleomycin-induced rat, Chin. J. Ind. Hygi. Occup. Dis. 4 (2010) 260263.

6. X. M. Li, J. H. Peng, Z. L. Sun, H. J. Tian, X. H. Duan, L. Liu, X. Ma, Q. Feng, P. Liu and Y. Y. Hu, Chinese medicine CGA formula ameliorates DMN-induced liver fibrosis in rats via inhibiting MMP2/9, TIMP1/2 and the TGF- $\beta / S m a d$ signaling pathways, Acta. Pharmacol. Sin. 6 (2016) 783-793; https://doi.org/10.1038/aps. 2016.35

7. E. Aghadavod, Amygdalin; is it an anticancer and antitumor agent? Epidemiol. Prev. 2 (2016) e22.

8. J. Q. Liu, D. Y. Wang and L. Wang, Influences of treatment methods on water absorption of Prunus mongolica seeds and its seedling growth, Chin. For. Sci. Technol. 21 (2010) 38-41.

9. Y. Q. Ma, Flora of Inner Mongolia, Vol. 3, Hohhot: Inner Mongolia People's Publishing House, 1989, p. 132.

10. S. L. Shi, Y. C. Bai, H. B. Zhou and S. F. Niu, Extraction and determination of content of polysaccharides in Amygdalus mongolica, Lishizhen Med. Mater. Med. Res. 24 (2013) 257-258.

11. K. Su, S. L. Shi, D. H. Zheng and J. X. Li, Determination of alpha tocopherol content in Amygdalus mongolica by HPLC, Chin. J. Exp. Trad. Med. Form. 19 (2013) 70-72.

12. J. Q. Guo, W. Z. Wu, M. X. Sheng, S. L. Yang and J. M. Tan, Amygdalin inhibits renal fibrosis in chronic kidney disease, Mol. Med. Rep. 5 (2013) 1453-1457; https://doi.org/10.3892/mmr.2013.1391

13. H. H. Luo, F. Zhao, F. X. Zhang and L. Ni, Influence of amygdalin on PDG, IGF and PDGFR expression in HSC-T6 cells, Exp. Ther. Med. 15 (2018) 3693-3698; https://doi.org/10.3892/etm.2018.5886

14. Y. S. Zhao, P. S. Wu, H. W. Zhang, X. H. Cheng, S. L. Shi, Q. L. Liu and H. B. Zhou, Studies on doseeffect relationship of n-butanol extracts of Amygdalus mongolica on reducing blood lipid and its chemical constituents, Sci. Technol. Food. Ind. 38 (2017) 348-352.

15. J. J. Jin, M. Zhong, S. M. Yu and L. Jie, Effect of water extract of Cudrania cochinchinensis on liver fibrosis rat models induced by carbon tetrachloride, Chin. J. Exp. Trad. Med. Formu. 22 (2012) 258-262.

16. M. P. Ren, Y. Liu, H. Li, M. H. Liu, H. Zhang and J. B. Wu, Study on Aralia saponins in preventing CCL4 induced liver fibrosis in rats, J. Shenyang Pharm. Univ. 12 (2013) 958-960.

17. S. F. Zhao and Q. C. Kan, Protective effects of Liuweiwuling pian on hepatic fibrosis induced by carbon tetrachloride in rats, Chin. Pharm. Bull. 6 (2011) 872-875; https://doi.org/10.3969/j.issn.10011978.2011.06.031

18. G. W. Newton, E. S. Schmidt, J. P. Lewis, E. Conn and R. Lawrence, Amygdalin toxicity studies in rats predict chronic cyanide poisoning in humans, West J. Med. 2 (1981) 97-103; https://doi.org/10.1620/ tjem.133.481

19. Y. A. Lee and S. L. Friedman, Reversal, maintenance or progression: What happens to the liver after a virologic cure of hepatitis C? Antiviral. Res. 107 (2014) 23-30; https://doi.org/10.1016/j.antiviral.2014.03.012

20. J. P. Li, Y. Gao, S. F. Chu, Z. Zhang, C. Y. Xia, Z. Mou, X. Y. Song, W. B. He, X. F. Guo and N. H. Chen, Nrf2 pathway activation contributes to anti-fibrosis effects of ginsenoside Rg1 in a rat model of alcohol- and $\mathrm{CCl}_{4}$-induced hepatic fibrosis, Acta. Pharmacol. Sin. 8 (2014) 1031-1044; https://doi. org/10.1038/aps.2014.41

21. S. Yamada and C. Hirayama, Clinical significance of serum hydroxyproline-containing peptides with special reference to hyproprotein, Eur. J. Clin. Invest. 2 (2010) 129-133; https://doi. org/10.1111/j.1365-2362.1983.tb00077.x

22. G. G. Xu, C. Y. Luo, S. M. Wu and C. L. Wang, The relationship between staging of hepatic fibrosis and the levels of serum biochemistry, Hepatobiliary Pancreat, Dis. Int. 2 (2002) 246-248; https://doi.org/ CNKI:SUN:GJGD.0.2002-02-019

23. S. P. You, J. Zhao, L. Ma, M. Tudimat, S. L. Zhang and T. Liu, Preventive effects of phenylethanol glycosides from Cistanche tubulosa on bovine serum albumin-induced hepatic fibrosis in rats, J. Pharm. Sci. 1 (2015) 1-13; https://doi. org/10.1186/s40199-015-0135-4 
24. J Liu, J. Y. Wang and Y. Lu, Serum fibrosis markers in diagnosing liver fibrosis, Chin. J. Intern. Med. 6 (2006) $475-477$.

25. S. Clichici, C. Catoi, T. Mocan, A. Filip, C. Login, A. L. Nagy, D. Doina, N. Decea, C. Gherman, R. Moldovan and A. Muresan, Non-invasive oxidative stress markers for liver fibrosis development in the evolution of toxic hepatitis, Acta Physiol. Hung. 98 (2011) 195-204; https:// 10.1556/APhysiol.98.2011.2.11

26. H. B. Tan, Q. He, R. G. Li, and F. F. Lei and X. Lei, Trillin Reduces Liver Chronic Inflammation and Fibrosis in Carbon Tetrachloride $\left(\mathrm{CCl}_{4}\right)$ Induced Liver Injury in Mice, Immunol. Commun. 45 (2016) 371-382; https://doi.org/10.3109/08820139. 2015.1137935

27. T. A. Wynn, Cellular and molecular mechanisms of fibrosis, J. Pathol. 2 (2010) 199-210; https://doi. org/10.1002/path.2277

28. C. J. Parsons, T. Motoki and R. A. Rippe, Molecular mechanisms of hepatic fibrogenesis, J. Gastroenterol. Hepatol. 1 (2007) S79-84; https://doi.org/10.1111/ j.1440-1746.2006.04659.x

29. Y. M. Yang and E. Seki, TNF $\alpha$ in liver fibrosis, Curr. Pathobiol. Rep. 4 (2015) 253-261; https://doi. org/10.1007/s40139-015-0093-z

30. A. Mehra and J. L. Wrana, TGF- $\beta$ and the Smad signal transduction pathway, Biochem. Cell Biol. 5 (2002) 605-622; https://doi.org/10.1139/o02-161

31. F. Y. Xu, C. W. Liu, D. D. Zhou and L. Zhang, TGF/SMAD Pathways and its regulation in hepatic fibrosis, J. Histochem. Cytochem. 3 (2016) 157-167; https:// doi.org/10.1369/0022155415627681 